\title{
Serum fragmented cytokeratin 18 levels reflect the histological activity score of nonalcoholic fatty liver disease more accurately than serum
}

\section{alanine aminotransferase levels}

Masaru Tsutsui, BE, ${ }^{\star}$ Naoki Tanaka, MD, PhD, ${ }^{\ddagger \S}$ Masatomo Kawakubo, PhD, , Yo

Sheena, DE, đ Akira Horiuchi, MD, PhD,\& Michiharu Komatsu, MD, PhD,§ Tadanobu Nagaya, MD,§ Satoru Joshita, MD,§ Takeji Umemura, MD, PhD,§ Tetsuya Ichijo, MD, $\mathrm{PhD}, \S$ Akihiro Matsumoto, MD, PhD, Kaname Yoshizawa, MD, PhD,§ Toshifumi Aoyama, $\mathrm{PhD},{ }^{\ddagger}$ Eiji Tanaka, $\mathrm{MD}, \mathrm{PhD}, \S$ and Kenji Sano, $\mathrm{MD}, \mathrm{PhD} \|$

Departments of Molecular Oncology ${ }^{\star}$ and Metabolic Regulation ${ }^{\ddagger}$, Institute on Aging and Adaptation, Shinshu University Graduate School of Medicine, §Department of Gastroenterology, Shinshu University School of Medicine, "Department of Laboratory Medicine, Shinshu University Hospital, TDepartment of Economics, Shinshu University, and \&Department of Gastroenterology, Showa Inan General Hospital

Corresponding author: Naoki Tanaka, $\mathrm{MD}, \mathrm{PhD}$

Department of Metabolic Regulation, Institute on Aging and Adaptation, Shinshu 
University Graduate School of Medicine, Asahi 3-1-1, Matsumoto, 390-8621, Japan

E-mail address: naopi@shinshu-u.ac.jp

Tel.:+81-263-37-2850

Fax: +81-263-37-3094

Tsutsui $\mathrm{M}$ and Tanaka $\mathbf{N}$ contributed equally to this work.

Conflicts of interest: The authors have declared that no conflict of interest exists.

Financial support: The authors have declared that no financial support exists.

Short title: Fragmented CK18 as a predictor of NAFLD activity score

Key words: cytokeratin 18; AST; ALT; NAFLD activity score

Abbreviations: ALT, alanine aminotransferase; AST, aspartate aminotransferase; BMI, body mass index; CK, cytokeratin; ELISA, enzyme-linked immunosorbent assay; HBV, hepatitis B virus; HCV, hepatitis C virus; Hsp, heat shock protein; HOMA-IR, homeostasis model assessment for insulin resistance; NAFLD, nonalcoholic fatty liver disease; NAS, NAFLD activity score; NASH, nonalcoholic steatohepatitis; ROS, reactive oxygen species; SD, standard deviation; US, ultrasonography. 


\section{Abstract}

Background \& Goals: Reliable non-invasive biomarkers to assess the histological activity of nonalcoholic fatty liver disease (NAFLD) have not been established. Since the frequency of Mallory bodies is known to be closely associated with disease severity, we hypothesized that serum levels of Mallory body-related proteins were correlated with NAFLD histological activity and evaluated this possibility. Study: Serum levels of total and fragmented cytokeratin (CK) 18, heat shock protein (Hsp) 70, Hsp90 $\alpha$, ubiquitin +1 , and $\mathrm{p} 38 \alpha$ at the time of liver biopsy were measured in 118 NAFLD patients and their association with histological findings and NAFLD histological activity score (NAS) was investigated. $\underline{\text { Results: }}$ Serum levels of both forms of CK18 and Hsp90 $\alpha$ were markedly higher in patients having nonalcoholic steatohepatitis (NASH) compared with non-NASH ones. Both forms of CK18 significantly correlated with degree of steatosis, lobular inflammation, and ballooning, and showed stronger positive correlations with NAS than serum aspartate and alanine aminotransferase (AST and ALT). Multiple regression analysis further revealed that fragmented CK18 and AST were effective predictors of NAS, with the former being the more definitive of the two $(P<0.001$ vs. 0.005$)$. In 20 NAFLD patients who received a follow-up biopsy, changes in fragmented CK18 levels, but not AST or ALT levels, closely paralleled those in NAS. 
Conclusions: These results establish the usefulness of fragmented CK18 measurement for assessing and monitoring the histological activity of NAFLD. 


\section{Introduction}

The prevalence of nonalcoholic fatty liver disease (NAFLD) is increasing worldwide. ${ }^{1,2}$ Nonalcoholic steatohepatitis (NASH) is the severe and progressive form of NAFLD and may develop into cirrhosis, hepatic failure, and hepatocellular carcinoma. ${ }^{3-5}$ Several pharmacologic treatments have been developed for NASH, which necessitate the development of appropriate means to monitor disease severity and evaluate therapeutic response.

At present, the activity of NAFLD is measured by typical histological findings, such as steatosis, hepatocyte ballooning, and lobular inflammation, as well as NAFLD histological activity score (NAS), all of which are calculated according to the histopathological criteria designated by the NASH Clinical Research Network. ${ }^{6}$ A liver biopsy is considered essential to estimate the activity of NAFLD, but its invasiveness and cost often limit the chances for biopsy. Additionally, since NASH livers are more heterogeneous than those of chronic hepatitis $\mathrm{C}^{7}$ sampling errors are prone to occur in percutaneous liver biopsies. Thus, assessment of NAFLD activity using biopsied specimens only may lead to insufficient evaluation.

Serum alanine aminotransferase (ALT) levels are regarded as a reliable biomarker of hepatocyte damage. However, discrepancies in serum ALT concentrations and 
histological activity have been documented in chronic hepatitis $\mathrm{C}$ patients. ${ }^{8}$ It has also been reported that maintenance of normal ALT levels does not guarantee non-progression of NASH. ${ }^{9}$ Therefore, the development of other non-invasive biomarkers to compensate for the shortcomings of serum ALT measurement is needed for NAFLD/NASH.

Mallory bodies and hepatocyte ballooning, two important hallmarks of NASH, are closely associated with disease severity and progression. ${ }^{3}$ These pathologies often coexist; it has been demonstrated that $81 \%$ of NASH patients with Mallory bodies have ballooned hepatocytes as well. ${ }^{6}$ When hepatocytes are chronically exposed to oxidative stress and toxic substances, such as acetaldehyde, they become ballooned, accumulate fat, show a disruption in the keratin intermediate filament network, and form Mallory bodies. ${ }^{10,11}$ A Mallory body is an irregular-shaped aggresome composed of abnormally-phosphorylated and cross-linked keratins, such as cytokeratin (CK) 8 and 18, and stress-induced proteins, including ubiquitin and heat shock proteins (Hsp) 70 and $90 .{ }^{11}$ These cytokeratins are hyperphosphorylated by $\mathrm{p} 38$ mitogen-activated protein kinase and phosphatidylinositol 3-kinase, among others. Microarray analysis using primary hepatocytes isolated from mice treated with diethyl-1,4-dihydro-2,4,6-trimethyl-3,5-pyridinedicarboxylate, an inducer of Mallory 
body formation in vivo, also uncovered that up-regulation of these proteins was responsible for Mallory body formation. ${ }^{12}$ Since hepatocytes containing Mallory bodies are susceptible to apoptosis, ${ }^{13}$ we hypothesized that levels of Mallory body-associated proteins released from hepatocytes into peripheral blood may be increased in NASH patients and change in accordance with disease activity. To explore this possibility, serum levels of CK18, Hsp90a, Hsp70, p38a, and ubiquitin+1 were determined in 118 biopsy-proven NAFLD patients by enzyme-linked immunosorbent assay (ELISA), and the relationship between the levels of these proteins and histological findings was examined. 


\section{Patients and Methods}

\section{Patients}

One-hundred and eighteen Japanese NAFLD patients who had been admitted to Shinshu University Hospital or affiliated hospitals between 2004 and 2006 for liver biopsy were evaluated. The possibility of NAFLD was determined by the following criteria: (1) persistently abnormal ALT levels (> $30 \mathrm{U} / \mathrm{L}$ ) for more than 3 months, (2) no consumption of alcohol or hepatotoxic drugs, (3) negative results for hepatitis B virus (HBV) surface antigen, high titer of HBV core antibody, anti-hepatitis C virus (HCV) antibody, and autoantibodies, such as anti-nuclear and anti-mitochondrial antibodies, (4) the absence of abnormal serum ceruloplasmin levels and transferrin saturation ratios, and (5) the presence of hepatic steatosis in abdominal ultrasonography (US). ${ }^{14}$ The final diagnosis of NAFLD was confirmed by liver histology.

Body height and weight were measured at admission and body mass index (BMI) was calculated. The presence of obesity was defined as having a BMI of more than 25 $\mathrm{kg} / \mathrm{m}^{2}$ based on criteria released by the Japan Society for the Study of Obesity. ${ }^{15}$ Patients were considered to be hypertensive if their systolic/diastolic pressure was greater than 140/90 $\mathrm{mmHg}$ or if they were taking anti-hypertensive drugs. Patients were considered to be diabetic if they had a fasting glucose level equal to or higher than 126 
$\mathrm{mg} / \mathrm{dL}$ or if they were taking insulin or oral hypoglycemic agents. Patients were considered to have hyperlipidemia if their fasting serum levels of cholesterol or triglycerides were equal to or higher than 220 or $150 \mathrm{mg} / \mathrm{dL}$, respectively, or if they were taking lipid-lowering drugs. ${ }^{16}$

\section{Routine laboratory examination}

Blood samples were obtained at the time of liver biopsy in a fasting state and regular examinations, such as complete blood counts and blood chemistries, were carried out using standard methods. All remaining sera were immediately frozen and kept at $-80^{\circ} \mathrm{C}$ until use. The homeostasis model assessment for insulin resistance (HOMA-IR) value was calculated as fasting glucose $(\mathrm{mg} / \mathrm{dL}) \times$ fasting insulin $(\mu \mathrm{U} / \mathrm{mL}) / 405 .{ }^{17}$

\section{Measurement of Mallory body-related protein concentrations}

Serum concentrations of Mallory body components were determined in duplicate by ELISA from samples obtained on the day of liver biopsy. All measurements were completed within 1 week of sample collection. Serum levels of total and fragmented CK18 were measured by an M65 ELISA kit and M30-Apoptosense ELISA kit, respectively (PEVIVA AB, Bromma, Sweden). Serum Hsp70 levels were analyzed by in-house ELISA as described by Njemini et al. ${ }^{18}$ The ELISA kits for Hsp90 $\alpha$ and p38 $\alpha$ were purchased from Assay Design (Victoria, British Columbia, Canada), and that for 
ubiquitin+1 was obtained from R\&D systems (Minneapolis, MN, USA).

\section{Histopathological examination}

Liver samples were obtained from two different sites in the same lobe using a 14-gauge needle by percutaneous US-guided or laparoscopy-assisted biopsy. ${ }^{19}$ For patients who underwent a follow-up biopsy, samples were obtained from the same lobe as the initial biopsy. The lengths of the samples obtained were all more than $1.8 \mathrm{~cm}$, and the mean number of portal tracts found in each sample was $11.3 \pm 2.9$. The specimens were immediately fixed in $10 \%$ neutral formalin, and sections were cut at $4-\mu \mathrm{m}$ thickness and stained by the hematoxylin and eosin or Masson trichrome method. Histological findings were assessed in a blinded fashion by an independent pathological expert (KS) and scored according to the staging/grading system proposed by Kleiner et al. ${ }^{6}$ As a minor modification, Mallory bodies were scored as none to rare (0), few (1), or many (2). NAS was calculated as the unweighted sum of the scores for steatosis (0-3), lobular inflammation (0-3), and ballooning (0-2), and ranged from 0 to 8 . The histological diagnosis of NASH was made according to the presence of hepatocyte ballooning in addition to macrovesicular steatosis.

\section{Ethics}

This study was approved by the ethics committee of Shinshu University School of 
Medicine and adheres to the principles of the Declaration of Helsinki. Informed consent was obtained from all patients.

\section{Statistical analysis}

Results were expressed as a number (percentage) or mean \pm standard deviation (SD).

Significance was analyzed by the chi-square test for categorical variables and the Mann-Whitney $U$ or Wilcoxon signed rank test for continuous variables. Correlation coefficients were calculated using Spearman's rank correlation analysis. To search for independent predictors of NAS, multiple regression analysis using the backward stepwise variable selection method was conducted. All statistical analyses were performed using SPSS software version 15.0 for Windows (SPSS Inc., Chicago, IL, USA). A $P$ value of less than 0.05 was considered to be statistically significant. 


\section{Results}

\section{Clinical and Histological Characteristics of Patients}

Clinical and histological features of 118 NAFLD patients are shown in Table 1 and 2, respectively. Approximately $60 \%$ of cases had obesity and the mean BMI value was $26.5 \mathrm{~kg} / \mathrm{m}^{2}$. According to histological findings, patients were divided into those having NASH $(n=105)$ or not $(n=13)$. Age, BMI, serum concentrations of $\gamma$-glutamyltransferase, cholesterol, glucose, and insulin, glycohemoglobin levels, and HOMA-IR values were significantly increased in NASH patients (Table 1). Mallory bodies were observed in $89(75 \%)$ patients (Table 2$)$.

\section{Serum Concentrations of Mallory Body-Related Proteins}

Serum concentrations of total CK18, fragmented CK18, and Hsp90 $\alpha$ were significantly increased in NASH patients compared with non-NASH ones (Table 1). On the other hand, there were no meaningful differences in the ratio of fragmented to total CK18 levels or serum levels of Hsp70, p38 $\alpha$, and unbiquitin+1 between the groups (Table 1).

\section{Relationship between Histological Findings and Laboratory Data}

Correlations between histological scores and laboratory data were examined. The degree of steatosis positively correlated with serum levels of both forms of CK18, Hsp90 $\alpha$, and ALT, in addition to platelet count (Table 3). The severity of lobular 
inflammation was correlated with 6 parameters except for platelet count, and especially with serum AST levels (Table 3). The frequency of ballooned hepatocytes was also correlated with both forms of CK18, Hsp90 $\alpha$, and AST levels, as well as with HOMA-IR values (Table 3). Interestingly, total CK18, fragmented CK18, and Hsp90 $\alpha$ levels had positive correlations with all NAS components, namely, steatosis, lobular inflammation, and ballooning. Furthermore, Mallory body scores were positively linked to fragmented CK18 and AST levels and HOMA-IR values, but negatively linked to platelet count (Table 3). Lastly, a significant inverse correlation was found between fibrosis stage and platelet count (Table 3).

\section{Relationship between NAS and Serum Markers}

We next evaluated whether our test parameters were correlated with NAS. Of the 6 parameters that were significantly correlated with NAS, serum fragmented CK18 levels showed the strongest positive correlation $(r=0.485, P<0.001)$ (Figure 1). Multiple regression analysis uncovered that both fragmented CK18 and AST were effective predictors of NAS, with fragmented CK18 being the more definitive of the two (Table 4).

\section{Correlation between Changes in NAS and Fragmented CK18 levels}

In the 118 NAFLD patients analyzed, 20 underwent follow-up biopsies and were 
summarized in Table 5. To explore the clinical applicability of fragmented CK18 measurement in the evaluation and monitoring of NAFLD activity, we examined the relationship between alterations in NAS and those in fragmented CK18 levels in these patients and compared them with those in AST and ALT levels. A reduction in NAS was observed in $13(65 \%)$ subjects (from $5.8 \pm 1.4$ to $3.8 \pm 1.3, P=0.001$ ). Of them, a remarkable NAS improvement of 3 points or more was detected in 3 patients; one stemmed from lifestyle correction and the others from treatment with highly-purified eicosapentaenoic acid. ${ }^{20}$ In the NAS-improved group, decreases in fragmented CK18, AST, and ALT levels were found in 12 (92\%), 12 (92\%), and 11 (85\%) patients, respectively. These decreases were significant for fragmented CK18 (from $305 \pm 225$ to $150 \pm 79 \mathrm{U} / \mathrm{L}, P=0.018$ ), AST (from $66 \pm 29$ to $38 \pm 11 \mathrm{U} / \mathrm{L}, P=0.001$ ), and ALT levels (from $86 \pm 70$ to $39 \pm 18 \mathrm{U} / \mathrm{L}, P=0.024$ ).

Conversely, NAS had deteriorated in $7(35 \%)$ patients at the time of the second biopsy (from $4.4 \pm 1.6$ to $6.3 \pm 1.7, P=0.016$ ). Corresponding increases in fragmented CK18 levels were observed in $6(86 \%)$ patients, but those in AST and ALT levels were found in only 2 (29\%) patients each. Although not statistically significant, fragmented CK18 levels tended to increase (from $90 \pm 42$ to $275 \pm 276 \mathrm{U} / \mathrm{L}, P=0.071$ ) in the NAS-deteriorated group, but not AST (from $74 \pm 37$ to $52 \pm 27 \mathrm{U} / \mathrm{L}, P=0.352$ ) or ALT 
levels (from $122 \pm 69$ to $85 \pm 63 \mathrm{U} / \mathrm{L}, P=0.345$ ).

Lastly, although changes in fragmented CK18 levels were correlated with those in NAS $(r=0.748, P<0.001)$, the same was not true for changes in AST $(r=0.428, P=$ $0.060)$ or ALT levels $(r=0.440, P=0.052)$ (Figure 2$)$. 


\section{Discussion}

Previous studies have revealed the usefulness of serum fragmented CK18 assays for differentiating NASH from NAFLD or predicting the presence of NASH. ${ }^{21-24}$ Recently, Diab et al. showed a significant positive correlation between serum levels of fragmented CK18 and NAS in 65 NAFLD patients who had undergone bariatric surgery. ${ }^{25}$ Although they also reported that fragmented CK18 levels were markedly decreased 6 months afterwards, it was inconclusive whether such a decrease actually reflected an improvement in NAS because of the lack of follow-up biopsies. In the current study, we demonstrated that serum fragmented CK18 levels were closely correlated with individual NAS components and overall NAS in 118 patients with biopsy-proven NAFLD. Multiple regression analysis revealed that fragmented CK18 levels were a strong predictor of NAS. Furthermore, we uncovered for the first time that the degree of changes in fragmented CK18 levels corresponded with those in NAS in NAFLD patients using evidence from paired biopsies. Therefore, these results not only corroborate previous observations, but also demonstrate the potential utility of fragmented CK18 as a reliable non-invasive biomarker to monitor disease activity and evaluate the therapeutic response of NAFLD patients.

Serum fragmented CK18 levels were strikingly higher in NASH patients than in 
non-NASH ones, which is consistent with previous reports. ${ }^{21,22}$ Since hepatocyte apoptosis is one of the key components involved in the progression of steatosis to steatohepatitis and caspase 3 is reported to be activated in NASH livers, ${ }^{26}$ it is conceivable that CK18 fragments, generated mainly by the activation of caspase 3, are increased in the sera of NASH patients. On the other hand, total CK18 is released from cells during necrosis. ${ }^{27}$ In this study, serum total CK18 levels were increased with fragmented CK18 levels, but the ratio of fragmented to total CK18 levels, which is an indicator of ascendancy of apoptotic cell death, ${ }^{27}$ did not differ between the NAFLD subgroups. Thus, we can speculate that an increase in total CK18 might also contribute to an increase in serum fragmented CK18 levels in patients with NASH. Additionally, since hepatic mRNA expression of CK18 has been reported to be up-regulated by oxidative stress in mice, ${ }^{28}$ augmentation of CK18 expression in NASH livers might be associated with the increases in serum levels of both forms of CK18.

An unexpected finding in this study was that serum fragmented CK18 levels showed the strongest correlation with degree of steatosis among the histological parameters. Overproduction of reactive oxygen species (ROS) and the ensuing augmentation of oxidative stress may occur in fat-engorged hepatocytes, since ROS are generated mainly through mitochondrial fatty acid $\beta$-oxidation, whose pathway is 
enhanced by an excess of fatty acids. ${ }^{29-32}$ Accumulation of fatty acids and lipid peroxides in hepatocytes may thus trigger activation of caspase 3 and promote cleavage of total CK18. ${ }^{29,33}$ A similar significant association between the extent of steatosis and fragmented CK18 levels has already been elucidated in patients with chronic hepatitis C. ${ }^{34}$

As far as we know, this is the first study to assess the relationship between histological findings in NAFLD and serum concentrations of Mallory body-related proteins. Not only fragmented CK18 levels, but also total CK18 and Hsp90 $\alpha$ levels, were correlated with both NAS and all individual NAS factors. This finding is in agreement with the established fact that the presence of Mallory bodies is an indicator of the severity of NASH. ${ }^{3,35,36}$

Platelet count, serum AST levels, and HOMA-IR values were also correlated with the stage of fibrosis, suggesting that these parameters may reflect the progression of fibrosis in NAFLD as well. Differently from a report by Diab et al., ${ }^{25}$ fragmented CK18 levels did not show a meaningful association with the stage of fibrosis in the current analysis. This disagreement might be explained by the different proportion of patients with advanced fibrosis (3\% in Diabs' study vs. $27 \%$ in the present study).

It is noteworthy that fragmented CK18 levels correlated with NAS more strongly 
than ALT levels. More importantly, changes in NAS were mirrored by those in fragmented CK18 levels, but not by those in ALT levels. To our knowledge, this is the first trial to demonstrate fragmented $\mathrm{CK} 18$ as a potentially superior biomarker for evaluating NAS. Serum ALT levels are conventionally believed to be a surrogate biomarker of hepatocyte injury and/or death. However, a recent study using methionine choline-deficient diet-fed mice demonstrated that an increase in serum ALT levels in NASH was attributable not to enhancement of hepatocyte necrosis/apoptosis or hepatitis, but rather to induction of hepatic ALT mRNA levels, ${ }^{37}$ and a clear discrepancy between the changes in NAS and those in serum ALT levels has been observed in another clinical trial for NASH. ${ }^{38}$ Our findings corroborate the idea that serum ALT levels are not always a reliable biomarker of NAFLD activity. Thus, we would like to propose determination of serum fragmented CK18 levels along with ALT levels for accurate assessment of therapeutic response in future NAFLD/NASH studies.

Clinically, this study was able to support the potential usefulness of fragmented CK18 measurement for distinguishing NASH from NAFLD. Serum levels of total CK18 and Hsp90 $\alpha$ and HOMA-IR values were also significantly increased in NASH patients, meaning evaluation of these parameters might help identify patients having NASH more efficiently than one marker alone. Further studies are required to verify it. 
In conclusion, this study established the usefulness of serum fragmented CK18 as a non-invasive, simple, and reliable biomarker that may complement the shortcomings of liver biopsy and serum ALT measurement. Although the number of patients in our longitudinal analysis was small, we believe that measurement of serum fragmented CK18 levels is a promising tool for monitoring disease status and assessing treatment response in patients with NAFLD. To confirm our observations, larger validation analyses and longitudinal prospective studies are needed. 


\section{Acknowledgements}

We thank Mr. Trevor Ralph for his editorial assistance. 


\section{References}

1. Ong JP, Younossi ZM. Epidemiology and natural history of NAFLD and NASH. Clin Liver Dis 2007;11:1-16.

2. Ono M, Saibara T. Clinical features of nonalcoholic steatohepatitis in Japan: evidence from the literature. J Gastroenterol 2006;41:725-732.

3. Matteoni CA, Younossi ZM, Gramlich T, et al. Nonalcoholic fatty liver disease: a spectrum of clinical and pathological severity. Gastroenterology 1999;116:1413-1419.

4. Shimada M, Hashimoto E, Taniai M, et al. Hepatocellular carcinoma in patients with non-alcoholic steatohepatitis. J Hepatol 2002;37:154-160.

5. Nagaya T, Tanaka N, Komatsu M, et al. Development from simple steatosis to liver cirrhosis and hepatocellular carcinoma: a 27-year follow-up case. Clin J Gastroenterol 2008;1:116-121.

6. Kleiner DE, Brunt EM, Van Natta M, et al. Design and validation of a histological scoring system for nonalcoholic fatty liver disease. Hepatology 2005;41:1313-1321.

7. Ratziu V, Charlotte F, Heurtier A, et al. Sampling variability of liver biopsy in nonalcoholic fatty liver disease. Gastroenterology 2005;128:1898-1906.

8. Sanai FM, Benmousa A, Al-Hussaini H, et al. Is serum alanine aminotransferase level 
a reliable marker of histological disease in chronic hepatitis $\mathrm{C}$ infection? Liver Int 2008;28:1011-1018.

9. Mofrad P, Contos MJ, Haque M, et al. Clinical and histologic spectrum of nonalcoholic fatty liver disease associated with normal ALT values. Hepatology $2003 ; 37: 1286-1292$.

10. Janig E, Stumptner C, Fuchsbichler A, et al. Interaction of stress proteins with misfolded keratins. Eur J Cell Biol 2005;84:329-339.

11. Zatloukal K, French SW, Stumptner C, et al. From Mallory to Mallory-Denk bodies: what, how and why? Exp Cell Res 2007;313:2033-2049.

12. Nan L, Dedes J, French BA, et al. Mallory body (cytokeratin aggresomes) formation is prevented in vitro by p38 inhibitor. Exp Mol Pathol 2006;80:228-240.

13. Kawahara H, Matsuda Y, Takase S. Is apoptosis involved in alcoholic hepatitis? Alcohol Alcohol 1994;29:113-118.

14. Tanaka N, Tanaka E, Sheena Y, et al. Useful parameters for distinguishing nonalcoholic steatohepatitis with mild steatosis from cryptogenic chronic hepatitis in the Japanese population. Liver Int 2006;26:956-963.

15. The Examination Committee of Criteria for 'Obesity Disease' in Japan, Japan Society for the Study of Obesity. New criteria for 'obesity disease' in Japan. Circ J 
2002;66:987-992.

16. Komatsu M, Yazaki M, Tanaka N, et al. Citrin deficiency as a cause of chronic liver disorder mimicking non-alcoholic fatty liver disease. J Hepatol 2008;49:810-820.

17. Tanaka N, Nagaya T, Komatsu M, et al. Insulin resistance and hepatitis $\mathrm{C}$ virus: a case-control study of non-obese, non-alcoholic and non-steatotic hepatitis virus carriers with persistently normal serum aminotransferase. Liver Int 2008;28:1104-1111.

18. Njemini R, Demanet C, Mets T. Comparison of two ELISAs for the determination of Hsp70 in serum. J Immunol Methods 2005;306:176-182.

19. Tanaka N, Ichijo T, Okiyama W, et al. Laparoscopic findings in patients with nonalcoholic steatohepatitis. Liver Int 2006;26:32-38.

20. Tanaka N, Sano K, Horiuchi A, et al. Highly purified eicosapentaenoic acid treatment improves nonalcoholic steatohepatitis. J Clin Gastroenterol 2008;42:413-418.

21. Wieckowska A, Zein NN, Yerian LM, et al. In vivo assessment of liver cell apoptosis as a novel biomarker of disease severity in nonalcoholic fatty liver disease. Hepatology 2006;44:27-33.

22. Yilmaz Y, Dolar E, Ulukaya E, et al. Soluble forms of extracellular cytokeratin 18 
may differentiate simple steatosis from nonalcoholic steatohepatitis. World $\mathbf{J}$ Gastroenterol 2007;13:837-844.

23. Tarantino G, Conca P, Coppola A, et al. Serum concentrations of the tissue polypeptide specific antigen in patients suffering from non-alcoholic steatohepatitis.

Eur J Clin Invest 2007;37:48-53.

24. Malik R, Chang M, Bhaskar K, et al. The clinical utility of biomarkers and the nonalcoholic steatohepatitis clinical research network liver biopsy scoring system in patients with nonalcoholic fatty liver disease. J Gastroenterol Hepatol 2009;24:564-568.

25. Diab DL, Yerian L, Schauer P, et al. Cytokeratin 18 fragment levels as a noninvasive biomarker for nonalcoholic steatohepatitis in bariatric surgery patients. Clin Gastroenterol Hepatol 2008;6:1249-1254.

26. Feldstein AE, Canbay A, Angulo P, et al. Hepatocyte apoptosis and fas expression are prominent features of human nonalcoholic steatohepatitis. Gastroenterology 2003;125:437-443.

27. Kramer G, Erdal H, Mertens H, et al. Differentiation between cell death modes using measurements of different soluble forms of extracellular cytokeratin 18. Cancer Res 2004;64:1751-1756. 
28. Gonsebatt ME, Del Razo LM, Cerbon MA, et al. Arsenite induced oxidative damage in mouse liver is associated with increased cytokeratin 18 expression. Arch Toxicol 2007;81:619-626.

29. Begriche K, Igoudjil A, Pessayre D, et al. Mitochondrial dysfunction in NASH: causes, consequences and possible means to prevent it. Mitochondrion 2006;6:1-28.

30. Browning JD, Horton JD. Molecular mediators of hepatic steatosis and liver injury. J Clin Invest 2004;114:147-152.

31. Aoyama T, Peters JM, Iritani N, et al. Altered constitutive expression of fatty acid-metabolizing enzymes in mice lacking the peroxisome proliferator-activated receptor $\alpha(\operatorname{PPAR} \alpha)$. J Biol Chem 1998;273:5678-5684.

32. Tanaka N, Moriya K, Kiyosawa K, et al. PPAR $\alpha$ activation is essential for HCV core protein-induced hepatic steatosis and hepatocellular carcinoma in mice. J Clin Invest 2008;118:683-694.

33. Feldstein AE, Werneburg NW, Canbay A, et al. Free fatty acids promote hepatic lipotoxicity by stimulating TNF-alpha expression via a lysosomal pathway. Hepatology 2004;40:185-194.

34. Seidel N, Volkmann X, Länger F, et al. The extent of liver steatosis in chronic hepatitis $\mathrm{C}$ virus infection is mirrored by caspase activity in serum. Hepatology 
2005;42:113-120.

35. Shimada M, Hashimoto E, Kaneda H, et al. Nonalcoholic steatohepatitis: risk factors for liver fibrosis. Hepatol Res 2002;24:429-438.

36. Angulo P, Keach JC, Batts KP, et al. Independent predictors of liver fibrosis in patients with nonalcoholic steatohepatitis. Hepatology. 1999;30:1356-1362.

37. Liu R, Pan X, Whitington PF. Increased hepatic expression is a major determinant of serum alanine aminotransferase elevation in mice with nonalcoholic steatohepatitis. Liver Int 2009;29:337-343.

38. Ratziu V, Giral P, Jacqueminet S, et al. Rosiglitazone for nonalcoholic steatohepatitis: one-year results of the randomized placebo-controlled Fatty Liver Improvement with Rosiglitazone Therapy (FLIRT) Trial. Gastroenterology 2008;135:100-110. 


\section{Figure legends}

Figure 1. Correlations between NAS and laboratory data in 118 biopsy-proven NAFLD patients

Correlation coefficients were calculated using Spearman's rank correlation analysis.

Figure 2. Correlations between changes in NAS and those in serum fragmented CK18, AST, and ALT levels in 20 NAFLD patients who received follow-up biopsies Correlation coefficients were calculated using Spearman's rank correlation analysis. 
Total CK18

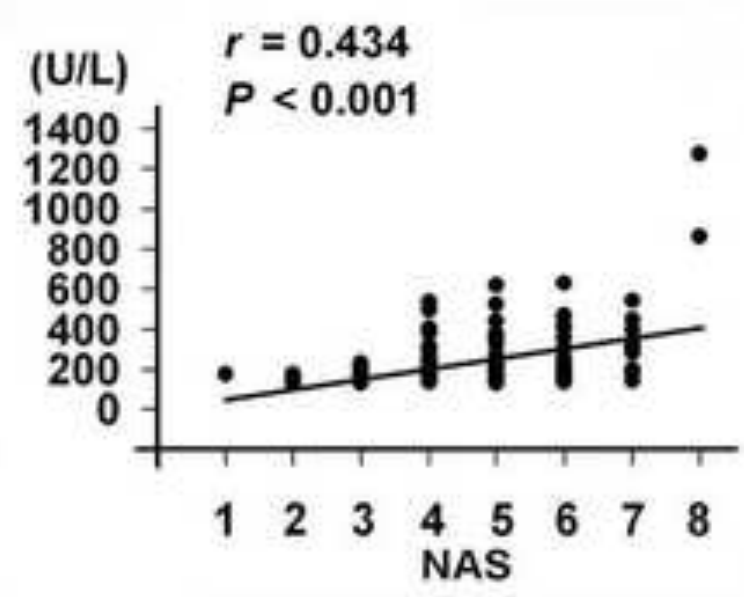

AST

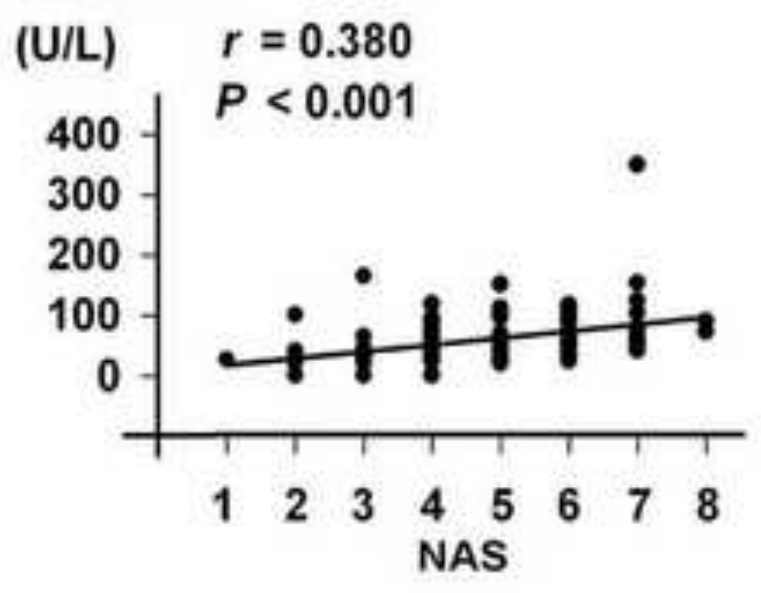

Fragmented CK18

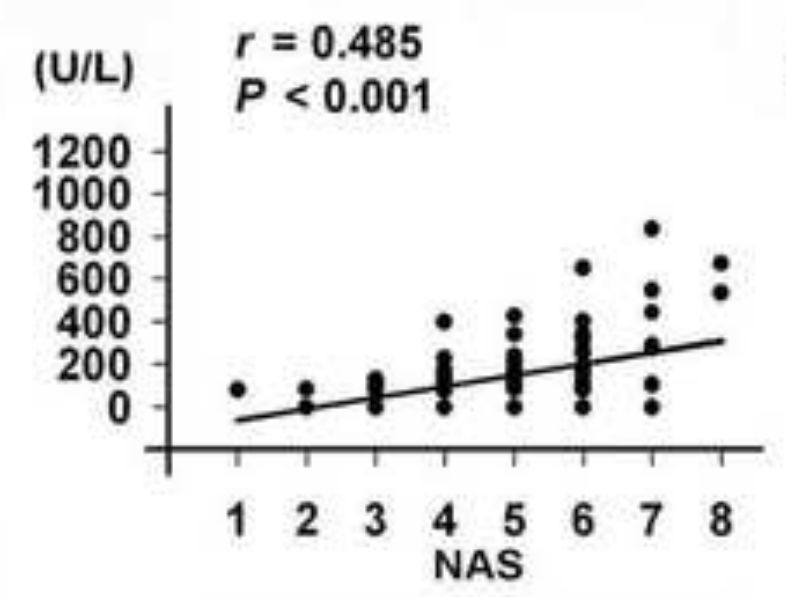

ALT

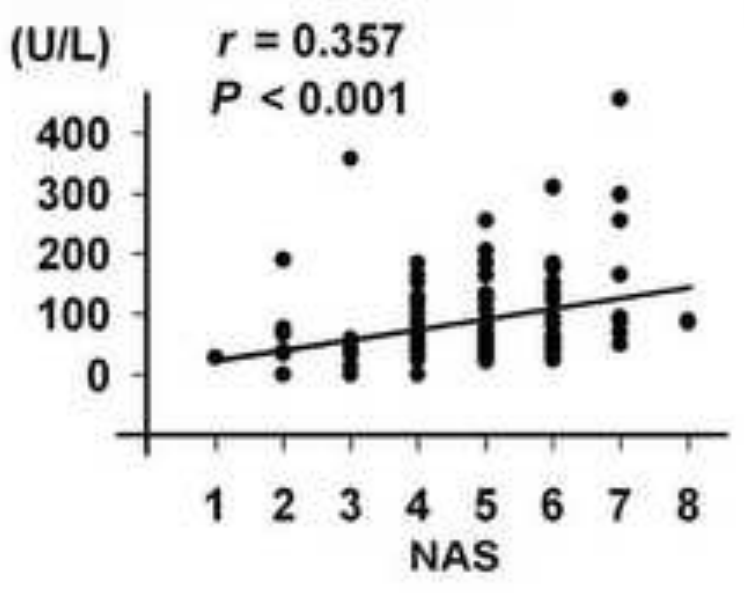

Hsp90a

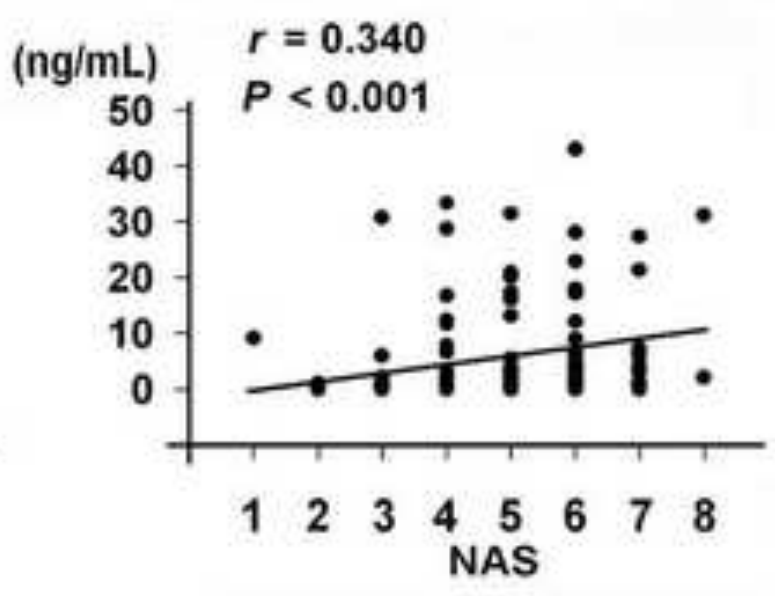

HOMA-IR

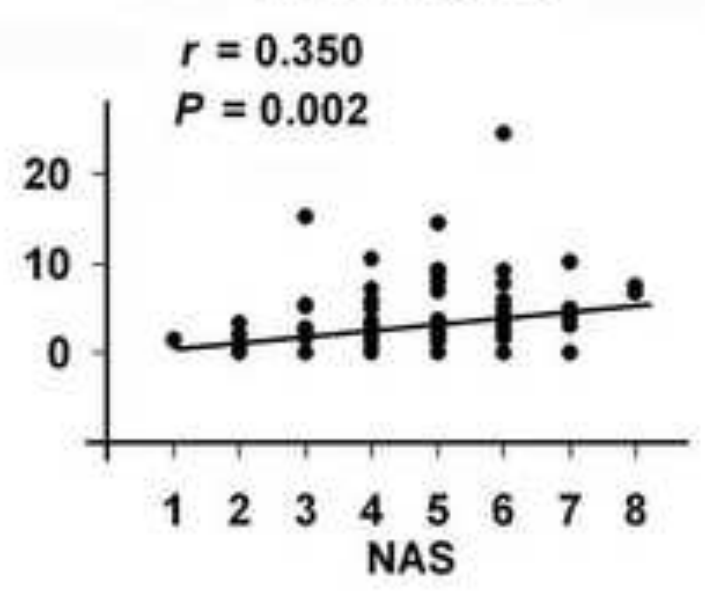



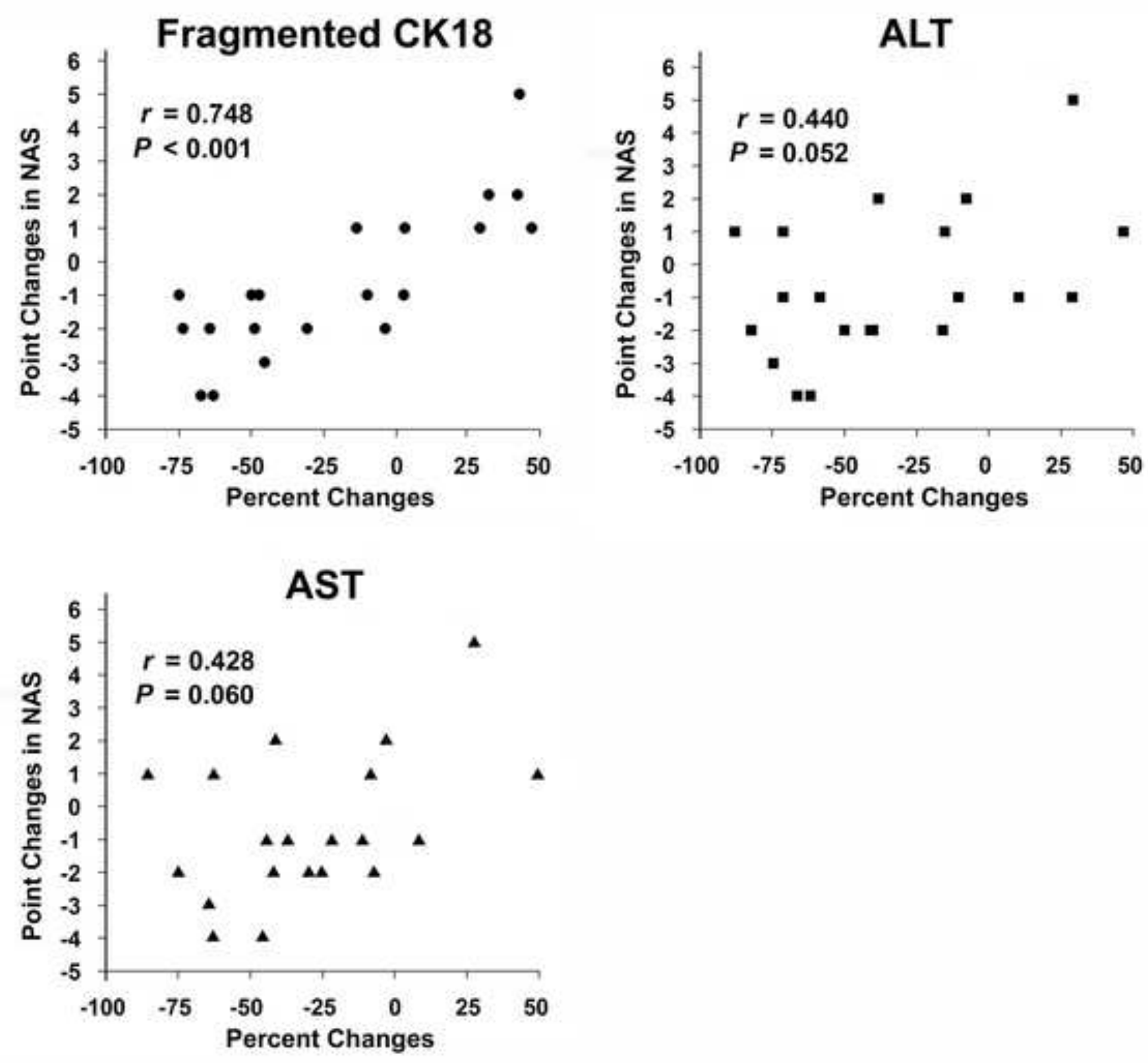
Table 1. Clinical features of 118 NAFLD patients

\begin{tabular}{|c|c|c|c|c|}
\hline & $\begin{array}{c}\text { Normal } \\
\text { values }\end{array}$ & $\begin{array}{c}\text { non-NASH } \\
(\mathrm{n}=13)\end{array}$ & $\begin{array}{c}\text { NASH } \\
(n=105)\end{array}$ & $P$ \\
\hline \multicolumn{2}{|l|}{ Age (yrs) } & $40 \pm 15$ & $57 \pm 16$ & $\underline{0.002}$ \\
\hline \multicolumn{2}{|l|}{ Female } & $3(\overline{23} \%)$ & $60(\overline{5} 7 \%)$ & $\overline{0.021}$ \\
\hline \multicolumn{2}{|l|}{ Obesity } & $4(31 \%)$ & $63(60 \%)$ & $\overline{0.046}$ \\
\hline \multicolumn{2}{|l|}{ Type 2 Diabetes } & $1(8 \%)$ & $38(36 \%)$ & $\overline{0.047}$ \\
\hline \multicolumn{2}{|l|}{ Hypertension } & $1(8 \%)$ & $42(40 \%)$ & $\overline{0.027}$ \\
\hline \multicolumn{2}{|l|}{ Hyperlipidemia } & $2(15 \%)$ & $41(39 \%)$ & $\overline{0.113}$ \\
\hline BMI $\left(\mathrm{kg} / \mathrm{m}^{2}\right)$ & $<25.0$ & $23.8 \pm 2.2$ & $26.9 \pm 3.9$ & $\underline{0.005}$ \\
\hline Platelet count $\left(\times 10^{3} / \mu \mathrm{L}\right)$ & $140-400$ & $222 \pm 77$ & $190 \pm 64$ & $\overline{0.189}$ \\
\hline C-reactive protein $(\mathrm{mg} / \mathrm{dL})$ & $<0.3$ & $0.2 \pm 0.2$ & $0.3 \pm 0.5$ & 0.596 \\
\hline AST (U/L) & $13-33$ & $46 \pm 29$ & $61 \pm 42$ & 0.072 \\
\hline ALT (U/L) & $8-42$ & $77 \pm 62$ & $92 \pm 76$ & 0.337 \\
\hline$\gamma \mathrm{GT}(\mathrm{U} / \mathrm{L})$ & $10-47$ & $115 \pm 61$ & $79 \pm 87$ & $\underline{0.038}$ \\
\hline Cholesterol (mg/dL) & $130-220$ & $178 \pm 33$ & $213 \pm 40$ & $\underline{0.005}$ \\
\hline Triglyceride (mg/dL) & $30-150$ & $140 \pm 55$ & $143 \pm 66$ & $\overline{0.961}$ \\
\hline LDL-cholesterol (mg/dL) & $<140$ & $86 \pm 0$ & $173 \pm 31$ & 0.143 \\
\hline Glucose (mg/dL) & $70-110$ & $84 \pm 9$ & $115 \pm 31$ & $\underline{0.001}$ \\
\hline Glycohemoglobin (\%) & $4.0-6.0$ & $4.9 \pm 0.4$ & $6.0 \pm 1.1$ & $\overline{\mathbf{0 . 0 1 3}}$ \\
\hline Insulin $(\mu \mathrm{U} / \mathrm{mL})$ & $2.0-15.0$ & $7.6 \pm 3.4$ & $14.9 \pm 10.6$ & $\underline{0.010}$ \\
\hline HOMA-IR & $<2.0$ & $1.6 \pm 0.8$ & $4.4 \pm 3.6$ & $\leq 0.001$ \\
\hline Ferritin (ng/mL) & $20-200$ & $277 \pm 203$ & $234 \pm 178$ & $\overline{0.404}$ \\
\hline Total CK18 (U/L) & & $159 \pm 34$ & $247 \pm 172$ & $\underline{0.041}$ \\
\hline Fragmented CK18 (U/L) & & $50 \pm 45$ & $143 \pm 153$ & $\overline{0.004}$ \\
\hline Fragmented CK18 / Total CK & & $0.4 \pm 0.3$ & $0.6 \pm 0.5$ & 0.387 \\
\hline Hsp90 $\alpha(\mathrm{ng} / \mathrm{mL})$ & & $1.3 \pm 2.6$ & $5.9 \pm 9.4$ & $\underline{0.041}$ \\
\hline Hsp70 (ng/mL) & & $0.4 \pm 1.5$ & $6.0 \pm 16.4$ & $\overline{0.117}$ \\
\hline $\mathrm{p} 38 \alpha(\mathrm{ng} / \mathrm{mL})$ & & $0.3 \pm 0.4$ & $0.5 \pm 0.7$ & 0.722 \\
\hline Ubiquitin+1 (ng/mL) & & $0.9 \pm 1.1$ & $0.7 \pm 1.4$ & 0.153 \\
\hline
\end{tabular}


One-hundred and eighteen biopsy-proven NAFLD patients were classified as those having NASH or not. Results are expressed as a number (percentage) or mean $\pm \mathrm{SD}$. Statistical analysis was performed using the chi-square test or the Mann-Whitney $U$ test. The $P$ values of less than 0.05 are bold and underlined. BMI, body mass index; AST, aspartate aminotransferase; ALT, alanine aminotransferase; $\gamma \mathrm{GT}, \gamma$-glutamyltransferase; LDL, low density lipoprotein; HOMA-IR, homeostasis model assessment for insulin resistance; CK, cytokeratin; Hsp, heat shock protein 
Table 2. Histological features of 118 NAFLD patients

\begin{tabular}{lccc}
\hline & $\begin{array}{c}\text { All } \\
(\mathrm{n}=118)\end{array}$ & $\begin{array}{c}\text { non-NASH } \\
(\mathrm{n}=13)\end{array}$ & $\begin{array}{c}\text { NASH } \\
(\mathrm{n}=105)\end{array}$ \\
\hline Steatosis & $0(0 \%)$ & $0(0 \%)$ & $0(0 \%)$ \\
0 & $50(42 \%)$ & $9(70 \%)$ & $41(39 \%)$ \\
1 & $41(35 \%)$ & $2(15 \%)$ & $39(37 \%)$ \\
2 & $27(23 \%)$ & $2(15 \%)$ & $25(24 \%)$ \\
3 & & & \\
Lobular inflammation & $41(35 \%)$ & $12(92 \%)$ & $29(28 \%)$ \\
$0-1$ & $55(47 \%)$ & $0(0 \%)$ & $55(52 \%)$ \\
2 & $22(19 \%)$ & $1(8 \%)$ & $21(20 \%)$ \\
3 & & & \\
Ballooning & & $13(100 \%)$ & $90(86 \%)$ \\
0 & $13(11 \%)$ & $0(0 \%)$ & $15(14 \%)$ \\
1 & $90(76 \%)$ & $0(0 \%)$ & \\
2 & $15(13 \%)$ & & $2(2 \%)$ \\
Fibrosis & & $7(54 \%)$ & $58(55 \%)$ \\
0 & $9(8 \%)$ & $4(31 \%)$ & $15(14 \%)$ \\
1 & $62(53 \%)$ & $0(0 \%)$ & $25(24 \%)$ \\
2 & $15(13 \%)$ & $2(15 \%)$ & $5(5 \%)$ \\
3 & $27(23 \%)$ & $0(0 \%)$ &
\end{tabular}




\begin{tabular}{|c|c|c|c|}
\hline \multicolumn{4}{|c|}{ Mallory body* } \\
\hline 0 & $29(25 \%)$ & $11(85 \%)$ & $18(17 \%)$ \\
\hline 1 & $57(48 \%)$ & $2(15 \%)$ & $55(52 \%)$ \\
\hline 2 & $32(27 \%)$ & $0(0 \%)$ & $32(31 \%)$ \\
\hline \multicolumn{4}{|c|}{ NAS } \\
\hline 1 & $1(1 \%)$ & $1(8 \%)$ & $0(0 \%)$ \\
\hline 2 & $7(6 \%)$ & $7(54 \%)$ & $0(0 \%)$ \\
\hline 3 & $16(14 \%)$ & $2(15 \%)$ & $14(13 \%)$ \\
\hline 4 & $32(27 \%)$ & $3(23 \%)$ & $29(28 \%)$ \\
\hline 5 & $28(24 \%)$ & $0(0 \%)$ & $28(27 \%)$ \\
\hline 6 & $22(19 \%)$ & $0(0 \%)$ & $22(21 \%)$ \\
\hline 7 & $10(9 \%)$ & $0(0 \%)$ & $10(9 \%)$ \\
\hline 8 & $2(2 \%)$ & $0(0 \%)$ & $2(2 \%)$ \\
\hline
\end{tabular}

The amount of Mallory bodies was divided into none to rare (0), few (1), or many (2). Results are expressed as a number (percentage). NAS, NAFLD activity score 
Table 3. Correlations between histological findings and laboratory data among 118 NAFLD patients

\begin{tabular}{lccccc}
\hline & Steatosis & $\begin{array}{c}\text { Lobular } \\
\text { inflammation }\end{array}$ & Ballooning & $\begin{array}{c}\text { Mallory } \\
\text { body }\end{array}$ & Fibrosis \\
\hline Platelet & $0.376^{* *}$ & - & - & $-0.250^{* *}$ & $-0.432^{* *}$ \\
AST & - & $0.471^{* *}$ & $0.205^{*}$ & $0.290^{* *}$ & $0.361^{* *}$ \\
ALT & $0.213^{*}$ & $0.355^{* *}$ & - & - & - \\
HOMA-IR & - & $0.362^{* *}$ & $0.311^{* *}$ & $0.353^{* *}$ & $0.376^{* *}$ \\
Total CK18 & $0.352^{* *}$ & $0.264^{* *}$ & $0.325^{* *}$ & - & - \\
Fragmented CK18 & $0.482^{* *}$ & $0.295^{* *}$ & $0.215^{* *}$ & $0.200^{*}$ & - \\
Hsp90 $\alpha$ & $0.272^{* *}$ & $0.194^{*}$ & $0.236^{*}$ & - & - \\
\hline
\end{tabular}

Correlation coefficients were calculated by Spearman's rank correlation analysis. AST, aspartate aminotransferase, ALT, alanine aminotransferase, HOMA-IR, homeostasis model assessment for insulin resistance; CK, cytokeratin; Hsp, heat shock protein; -, not significant; *, $P<0.05 ; * *, P<0.01$ 
Table 4. Selected results of multiple regression analysis

\begin{tabular}{lcccc}
\hline & $\begin{array}{c}\text { Partial } \\
\text { regression } \\
\text { coefficient }\end{array}$ & $\begin{array}{c}\text { Standardized partial } \\
\text { regression } \\
\text { coefficient }\end{array}$ & $P$ & $\begin{array}{c}95 \% \\
\text { confidence } \\
\text { interval }\end{array}$ \\
\hline Fragmented CK18 & 0.004 & 0.442 & $<0.001$ & $0.002-0.006$ \\
AST & 0.010 & 0.287 & 0.005 & $0.003-0.016$ \\
\hline
\end{tabular}

Multiple regression analysis was performed using the backward stepwise variable selection method. Serum levels of aspartate aminotransferase (AST), alanine aminotransferase, total and fragmented cytokeratin (CK) 18, and heat shock protein $90 \alpha$, as well as homeostasis model assessment for insulin resistance values were selected as independent variables. Multiple correlation coefficient and coefficient of determination were 0.550 and 0.302 , respectively. 
Table 5. Characteristics of 20 patients who received a follow-up biopsy

\begin{tabular}{lc}
\hline Characteristics & Values \\
\hline Age (yrs) & $61 \pm 15$ \\
Female & $13(65 \%)$ \\
NAS at first biopsy & $5.3 \pm 1.6$ \\
Interval between biopsies (yrs) & $2.9 \pm 1.4$ \\
Intervention & \\
$\quad$ Lifestyle correction & $8(40 \%)$ \\
Treatment with highly-purified EPA & $8(40 \%)$ \\
Treatment with pioglitazone & $3(15 \%)$ \\
Treatment with bezafibrate & $1(5 \%)$ \\
Changes in NAS & $13(65 \%)$ \\
Improved & $0(0 \%)$ \\
Unchanged & $7(35 \%)$ \\
Deteriorated &
\end{tabular}

NAS, NAFLD activity score; EPA, eicosapentaenoic acid 